\title{
Latarjet Procedure for the Treatment of Anterior Glenohumeral Instability in the Athlete - Key Considerations for Rehabilitation
}

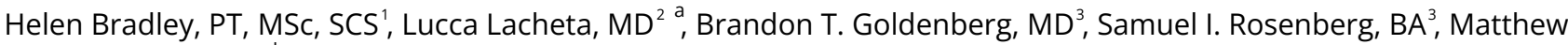 \\ T. Provencher, $\mathrm{MD}^{4}{ }^{\mathrm{b}}$, Peter J. Millett, MD, $\mathrm{MSc}^{4} \mathrm{c}$ \\ ${ }^{1}$ Howard Head Sports Medicine, ${ }^{2}$ Steadman Philippon Research Institute; Charité-Universitätsmedizin Berlin, ${ }^{3}$ Steadman Philippon Research \\ Institute, ${ }^{4}$ Steadman Philippon Research Institute; The Steadman Clinic \\ Keywords: shoulder, return to sport, rehabilitation, movement system, latarjet \\ https://doi.org/10.26603/001c.18707
}

\section{International Journal of Sports Physical Therapy}

Vol. 16, Issue 1, 2021

\begin{abstract}
The Latarjet procedure with transfer of the coracoid process and its attached conjoint tendon is a well-established surgical technique for the treatment of anterior glenohumeral instability in patients with anteroinferior bone loss and/or high risk for recurrence. Biomechanical and clinical studies have shown excellent results and high rates of return to sports. However, there is an absence of standardized, objective criteria to accurately assess an athlete's ability to progress through each phase of rehabilitation. Return to sports rehabilitation, progressed by quantitatively measured functional goals, may improve the athlete's integration back to sports participation. Therefore, the purpose of this clinical commentary is to provide a rehabilitation protocol for the Latarjet procedure, progressing through clearly defined phases, with guidance for safe and effective return to sport. Recommended criteria are highlighted which allows the clinician to progress the patient through each phase appropriately rather than purely following timeframes from surgery. This progression ensures the patient has completed a thorough rehabilitation program that addresses ROM, strength, power, neuromuscular control and a graded return to play.

Level of Evidence: 5
\end{abstract}

\section{INTRODUCTION AND BACKGROUND}

The glenohumeral joint (GHJ) is the most commonly dislocated joint in the body, with over $90 \%$ of dislocations occurring anteriorly. ${ }^{1} \mathrm{GHJ}$ dislocation is especially common in young male athletes, with a high prevalence of $3 \%$ per year in this population. ${ }^{2,3}$ The greatest rates occur in contact sports, such as football, ice-hockey, and wrestling, or sports that may involve falls such as skiing, volleyball and gymnastics. ${ }^{4}$ In cases of anterior glenoid bone loss of $13.5 \%$ or greater, poor clinical outcomes have been reported after soft tissue repair ${ }^{5-7}$ thus indicating a bone augmentation procedure may be necessary in these circumstances. The Latarjet procedure is a possible surgical procedure to treat patients with anterior shoulder instability and accompanied bone loss. It involves transferring the coracoid process and its attached conjoint tendon to the anterior glenoid rim. Many authors describe excellent clinical outcomes, return to sport rates, and low rates of recurrence after Latarjet. ${ }^{8-13}$ Nonetheless, the Latarjet procedure results in significant distortion in normal anatomy and may be associated with a significant restriction in postoperative range of motion, ${ }^{14-16}$ a result that has been linked with high rates of osteoarthritis. ${ }^{17}$ It has been suggested that with early skilled physical therapy these restrictions could be avoided. ${ }^{18}$ The purpose of this clinical commentary is to provide a rehabilitation protocol for the Latarjet procedure, progressing through clearly defined phases, with guidance

\footnotetext{
a Dr. Lacheta's position at the Steadman Philippon Research Institute was supported by Arthrex.

b Dr. Provencher reports grants, personal fees and other from Arthrex, from Smith \& Nephew, from Siemens, from Össur, from Joint Restoration Foundation, from SLACK Incorporated, outside the submitted work.

c Corresponding Author:

Peter J. Millett, MD, MSc

Steadman Philippon Research Institute

The Steadman Clinic, 181 West Meadow Drive, Suite 400, Vail, CO 81657

Telephone: 970-479-5876, Fax: 970-479-9753

drmillett@thesteadmanclinic.com
}

Dr. Millett reports grants, personal fees and other from Arthrex, from Smith \& Nephew, from Siemens, from Össur, from Medibridge, from Springer Publishing, from VuMedi, outside the submitted work. 
for safe and effective return to sport.

\section{ANATOMY}

Unlike the hip joint where the femoral head sits completely in the acetabulum, the humeral head sits on a shallow glenoid fossa, with only $30 \%$ in contact with the glenoid in various shoulder positions. ${ }^{19-21}$ The GHJ has the highest range of motion of any joint in the human body and relies mainly on soft-tissue stability in the absence of adequate bony coverage. Its biomechanical stability depends on the interaction between static stabilizers and dynamic stabilizers. ${ }^{19}$ Static stabilizers include the articular geometry and congruence of the joint, the glenoid labrum, and the capsuloligamentous complex, which consists of the coracohumeral ligament, and the superior, middle, and inferior glenohumeral ligaments. Dynamic stabilizers of the GHJ include the scapulothoracic musculature, which include the trapezius, serratus anterior, levator scapulae, rhomboid major, and pectoralis minor, and the rotator cuff muscles, which include the supraspinatus, infraspinatus, teres minor, and subscapularis. The rotator cuff muscles, along with the long head of the biceps increase stability by a process called concavity compression, where muscle contraction forces the articular surfaces of the humerus and glenoid together. The periscapular muscles maintain stability by controlling the position of the scapula during motion of the humerus. Disruption of any of these stabilizers can affect the inherent force couples and compromise stability. ${ }^{22}$ There is an intricate balance between static and dynamic stabilizers in order to provide glenohumeral stability. Rehabilitation principles should focus on maintaining this balance between static and dynamic stabilizers with overall goals of preserving motion and maintaining function and stability.

\section{BIOMECHANICS}

In 1954, Latarjet first proposed the transfer of the coracoid tip by suggesting that the horizontal limb of the coracoid process be fixed to the anteroinferior margin of the glenoid with a screw. ${ }^{23}$ The success of the intervention can be explained by a combination of effects: Firstly, the conjoint tendon acts as a sling to the inferior subscapularis and anteroinferior capsule when the arm is abducted and externally rotated. ${ }^{24}$ Second, the addition of bone to the glenoid rim increases the anteroposterior (AP) osseous diameter. ${ }^{25}$ Third, the inferior capsule is reinforced with a portion of the coracoacromial ligament. ${ }^{24}$ Cadaveric biomechanical studies have concluded that it is the combination of the bone block, sling, and capsule effect that are helpful in preventing abnormal translation and enhancing stability. Payne et al showed that the combination of the conjoint tendon and coracoid transfer was significantly superior in increasing stability compared to conjoint tendon transfer alone. ${ }^{26}$ Wellman et al. attributed a significant decrease in translation at $60^{\circ}$ of abduction to the sling effect or capsular repair as opposed to just the bone block. ${ }^{27}$ Yamamoto et al. evaluated the contribution to stability of the bone block, sling, and capsule repair and concluded that the sling effect provided $76-77 \%$ and capsule $23-24 \%$ of the stability at the end-range arm position and the sling contributed $51-62 \%$ and the bone block $38-49 \%$ at the mid-range position. ${ }^{28}$ This is known as the triple blocking effect of the Latarjet procedure, and it should be noted that each portion of the procedure contributes to the overall stability of the GHJ.

\section{SURGICAL TECHNIQUE}

A standard deltopectoral approach is used to expose the glenohumeral joint and the coracoid process medially. Next, the pectoralis minor is incised off the medial aspect of the coracoid and the coracoacromial ligament is excised off the lateral aspect. Once the coracoid is freed from circumferentially attached soft tissue with preserved conjoint tendon anteriorly, an oscillating saw is utilized to osteotomize the coracoid in a superior to inferior and medial to lateral trajectory. The graft length is on average $25 \mathrm{~mm}$ in length. Subsequently, the undersurface of the coracoid is decorticated with a saw, and two drill holes are placed through the coracoid graft.

Next, a subscapularis tendon and capsule split at the junction of the inferior and middle third of the tendon in line with the fibers is performed. Then, a decortication of the anterior aspect of the glenoid neck down to a bleeding bone surface to enhance bone-to-bone healing is done. Two drill holes are established in anterior to posterior direction for later fixation of the coracoid graft to the glenoid. Two $3.5 \mathrm{~mm}$ screws are used to reduce the coracoid block and construct stability will be confirmed (igure 1).

\section{POSTOPERATIVE REHABILITATION}

A successful outcome following Latarjet surgery is a painfree, stable shoulder that has enough mobility, stability and strength for a patient's desired level of activity. Additionally, improving function and reducing long-term sequelae is essential.

Literature to support the efficacy of a specific rehabilitation protocol for Latarjet reconstruction is limited. Gaunt et al. developed a consensus rehabilitation guideline following arthroscopic anterior capsulolabral repair and reported initial rehabilitation should aim to restore motion, strength and basic function, while protecting the surgical procedure. ${ }^{18}$ The available evidence and guidelines are less clear for the later stages of rehabilitation and determination regarding return to sports.

It is the intention of this clinical commentary to describe a post-operative rehabilitation protocol following a Latarjet procedure and introduce a criterion-driven algorithm for progression through return to sport rehabilitation.

\section{PHASE I: PROTECTION PHASE}

The goals of this phase of rehabilitation are to protect the surgical reconstruction, optimize the environment for tissue healing, control edema and swelling, and achieve protected range of motion (ROM). These goals must be addressed without causing inappropriate stress to the healing structures. 
Patient education to convey the rationale of the outlined plan of care and the importance of protecting the healing tissues from excessive stress is critical to ensure compliance. In order to protect the reconstruction, the arm is immobilized for four weeks in an abduction sling. Early passive ROM (PROM) exercises are beneficial in preventing postoperative stiffness. Due to the violation of the subscapularis tendon and repair of the capsule, external rotation (ER) should be limited to $<30^{\circ}$ for the first three weeks. ${ }^{29}$ No limitations on other ranges are needed and progression to full PROM is recommended early. In addition to PROM, manual therapy during this phase should consist of soft tissue techniques such as myofascial techniques for the shoulder musculature, specifically the pectorals, as well as scar mobilization. Cryotherapy and other modalities to decrease swelling and muscle spasm and aid in pain relief and sleep are also advocated as needed.

Supplemental activities include active scapular exercises (protraction, retraction, elevation and depression), such as the scapular clock exercise, to facilitate early neuromuscular control. Active ROM of uninvolved joints (hand, wrist, elbow and neck) to minimize stiffness in surrounding joints and encourage blood flow. To protect the healing structures from shear or compressive forces, no isometric exercises should be performed during weeks $0-2$ following Latarjet procedure. Especially important is refraining from loading during elbow flexion and supination, to avoid pulling off the coracoid bone block from the glenoid.

After two weeks, isometric exercises of the muscles surrounding the shoulder girdle can be initiated. Submaximal, non-painful, isometrics in neutral rotation can be performed. ${ }^{30}$ Rhythmic stabilization (RS), involving gentle manual resistance to the proximal forearm using oscillating perturbation in a neutral shoulder position, while the patient is instructed to keep their arm still, are appropriate to facilitate low contraction of the scapular and rotator cuff musculature and can also be beneficial for early neuromuscular re-education and improving dynamic stability.

The criterion to progress to the next phase of rehabilitation is shown in Appendix 1.

\section{PHASE II: ENDURANCE PHASE}

The goals of this phase of rehabilitation are to advance active shoulder motion and improve muscular endurance and neuromuscular control of the shoulder complex. This should start with active-assisted ROM (AAROM) then progress to active ROM (AROM). AROM can be started in supine, side-lying or prone positions to reduce the effects of gravity, progressing to standing as endurance improves. It is recommended that isotonic exercises should focus on high volume training with $15-25$ repetitions, $2-3$ sets, with low resistance at less than $65 \%$ of the patients one repetition maximum (1RM) in order to create a fatigue response and target the development of local muscular endurance. ${ }^{31,32}$ Rest times between sets should be no more than $30 \mathrm{sec}-$ onds. ${ }^{31} \mathrm{ER}$ will often be slow to progress, but it is important to attain full ER ROM, as any deficit in ROM can have long term implications on joint health and development of osteoarthritis. ${ }^{15,33}$

The dynamic stabilizers of the shoulder provide stability

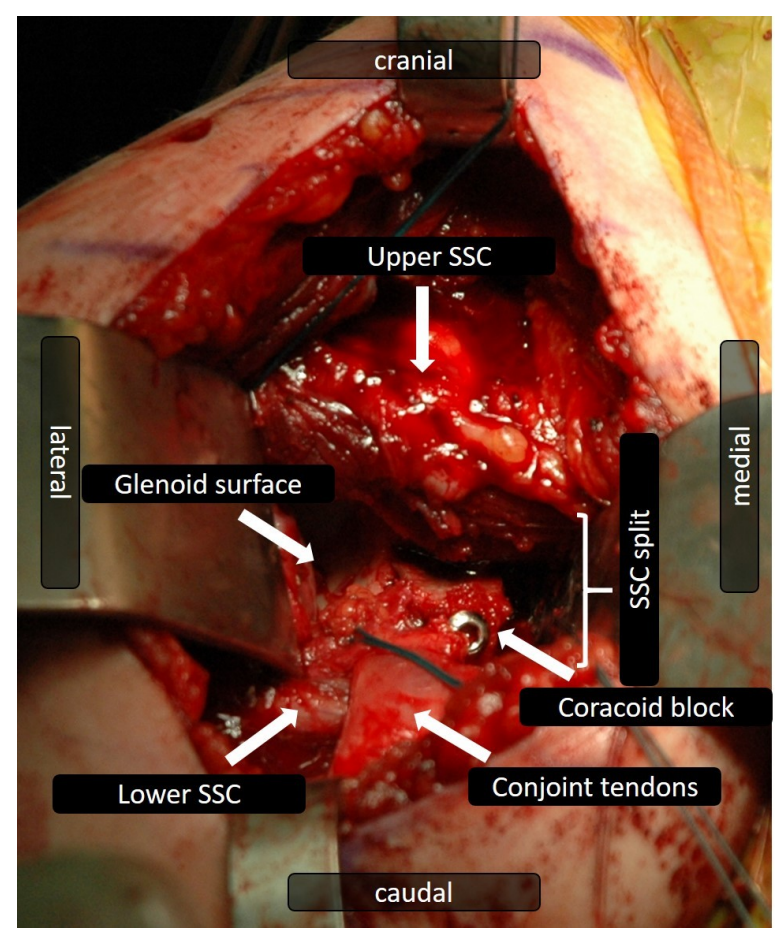

Figure 1. Deltopectoral approach to a right glenohumeral joint: A subscapularis (SSC) split was performed in the lower third to access the anterior glenoid. The coracoid tip was detached and transferred to the anteroinferior glenoid rim. The attached conjoint tendons are diverted through the subscapularis split.

through an active mechanism referred to as neuromuscular control. The development of neuromuscular control and proprioception should be a priority and begin as active ROM allows. Meyers and Lephart define neuromuscular control as the efferent output in response to afferent sensory (proprioceptive) stimulation. ${ }^{34}$ It can be assessed and enhanced through joint position sense (JPS) where the patient works on recognizing where one's joint is oriented in space. This can be limited in individuals with glenohumeral instability and not fully restored until 12 months after surgery for instability. ${ }^{35}$ An example of an exercise to address this deficit includes the patient closing his or her eyes, and moving the uninvolved upper extremity to a selected position and then actively moving their involved limb to the same position (Figure 2). The joint angular replication test (JART) can be used as an exercise as well as an assessment tool for JPS. ${ }^{36}$ This involves the rehabilitation specialist passively moving the involved limb to a position, the individual holds the position for spatial orientation then it is passively moved back to neutral. The patient actively moves the arm to as close to that position and this angle is measured with a goniometer or inclinometer. Normative data for healthy individuals resulted in an average error of 2.7 degrees. ${ }^{37}$ Movements can be practiced and tested in the sagittal, frontal and transverse planes.

Proprioceptive neuromuscular facilitation (PNF) exercises, such as D2 flexion/extension, should be started to help gain stability and control into functional patterns. Ad- 
vancing RS exercises towards end ROM and specifically for internal and external rotators will help facilitate co-contraction of the anterior and posterior rotator cuff musculature. 38

Scapular control should be advanced in this phase as it will help athletes optimize functional performance via improved kinetic chain integration. When the trapezius and the serratus anterior (SA) activate in unison and achieve their optimal force coupling pattern, they significantly contribute to scapular stability. ${ }^{39}$ Particular exercises which have been shown to accentuate peri-scapular control are prone horizontal abduction at $90^{\circ}$ with ER, prone extension exercise and prone overhead arm raise at $125^{\circ}$. Dynamic hugs and wall slides (Figure 3 ) have been shown to produce good SA activation. ${ }^{40}$

Supplemental techniques during this phase include soft tissue mobility and long duration stretching to address any persistent limitations in ROM. Gentle glenohumeral joint mobilizations can be initiated at five to six weeks post- operatively if mobility is slow to return.

The suggested criterion to progress to Phase III of rehabilitation is shown in Appendix 1. It is important that the patient has good neuromuscular control and muscular endurance to progress into the strengthening phase. The JART below $90^{\circ}$, the scapular dyskinesis test as described by McClure and colleagues, ${ }^{41}$ and the AROM fatigue protocol test are used to assess this. It is important to not have compensatory movement patterns with these tests.

Utilizing the lower extremity (LE) Y-Balance ${ }^{\mathrm{TM}}\left(\mathrm{Y}^{\mathrm{B}}-\mathrm{BAL}^{\mathrm{TM}}\right)$ assessment or similar test to identify any LE asymmetries or deficits should be performed at this time.

\section{PHASE III: STRENGTH PHASE}

The primary goals of Phase III are to increase muscular strength and challenge neuromuscular control. Individuals should not start this phase until Phase II goals are met and at least six weeks post-operative in order to ensure adequate tissue healing. All parameters of progression should also be communicated with all members of the treatment team. At this time, a thorough review of the athlete's sport requirements is beneficial. If the individual is an overhead athlete, progressing strength into the necessary ranges is important, as well as starting to replicate functional activities involved with their sport.

The strengthening parameters in this phase should start with loads which correspond to a repetition range of an 8-12 repetition maximum (RM) for 3-6 sets. ${ }^{42,43}$ These guidelines allow for a safe increase in load of the shoulder joint and are sufficient to create muscular adaptations. ${ }^{43}$ External load can be added to therapeutic exercises in the form of manual resistance from the rehabilitation specialist, dumbbells, medicine balls, kettle bells, sports cords and TheraBand $^{\mathrm{TM}}$. Most exercises early on in this phase will focus on strengthening specific muscle groups, such as shoulder external rotators or shoulder internal rotators. Once baseline strength has been established in each muscle group, functional movements with the involved upper extremity will start to replicate movement patterns involved in day to day activity as well as sports performance. De Mey and colleagues ${ }^{44}$ have demonstrated maximal scapular

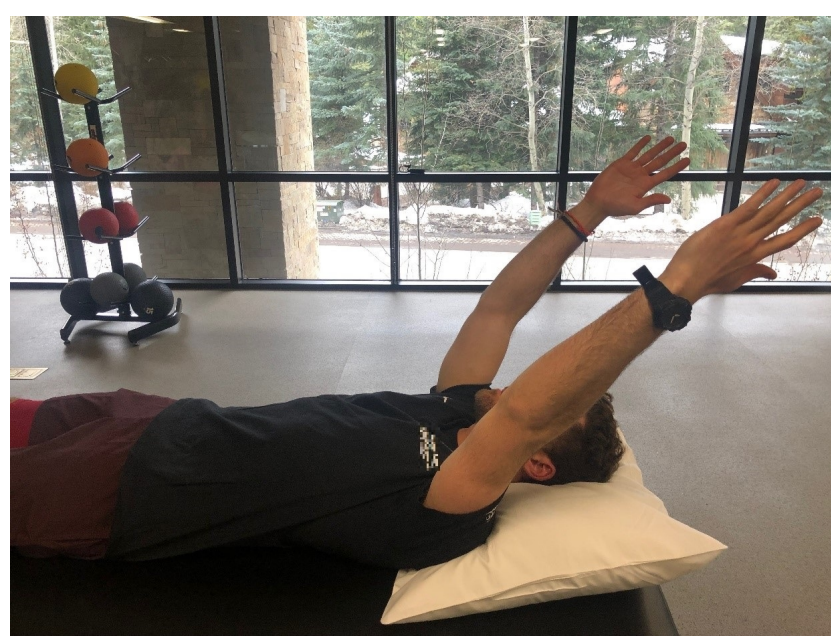

Figure 2. Joint position sense exercise: Patient in supine position with closed eyes. First, the uninvolved extremity is actively moved, then the affected extremity is moved to as close to that position as the uninvolved extremity.

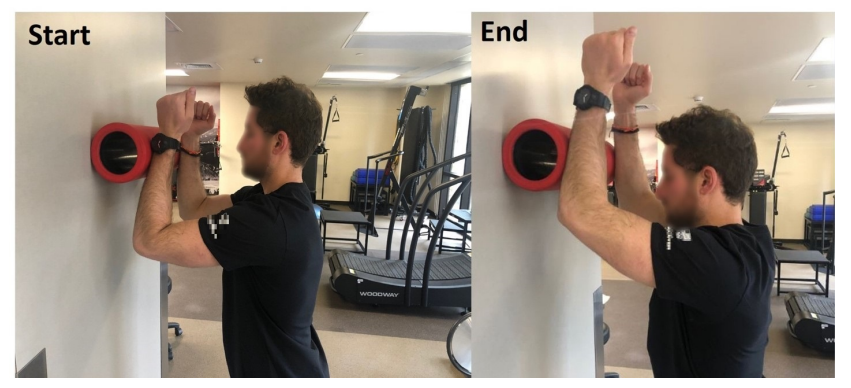

Figure 3. Wall slides to help activate the serratus anterior.

muscle activation when muscles are activated in functional patterns and when muscles are activated in specific diagonal patterns using kinetic chain sequences. Examples include resisted PNF exercises (Figure 4) and Crossover Symmetry exercises.

Dynamic stability can be enhanced through progression of neuromuscular control techniques, including reactive neuromuscular control drills and closed kinetic chain (CKC) exercises. RS exercises at this phase can be incorporated during performance of any exercise throughout the ROM. Placing an unstable base under the upper limb such as a stability ball enhances neuromuscular control. Adding perturbation to the exercise challenges proprioception and improves joint position. ${ }^{45} \mathrm{CKC}$ exercises stress the joint in a weight-bearing position, resulting in joint approximation. Advancing from wall to tabletop or to quadruped position (Figure 5) creates a gradual increase in loading and is excellent for scapular control. ${ }^{46,47}$ Additionally, performing exercises that introduce chaos can be beneficial for increasing core stability and rotator cuff recruitment. An example would be chaos band training, such as performing an overhead press with added weight suspended to the barbell, us- 
ing looped bands. This would add instability and unpredictability to the exercise.

It is critical to build the right balance when it comes to scapular foundation muscles, rotator cuff control, and transfer of energy from the legs, core and shoulder to the arm. The kinetic chain model refers to the ability to transfer large forces through segmental links in the body with the theory of gaining proximal stability for distal mobility, power and precision, all attributes that are required in sporting performance. ${ }^{48}$ Rehabilitation exercises that include integration of ipsilateral and contralateral kinetic activation in single leg squat, split squat, half and tall kneeling positions while adding distal resistance should be utilized in this phase.

Criterion to progress to the next phase is shown in Appendix 1 . There should be no apprehension on clinical evaluation. If the athlete is returning to an overhead sport it is important to consider their total arc of motion (TAOM). It is normal for an overhead athlete to have an increase in ER and a subsequent decrease in IR on their dominant side. The TAOM is the combination of ER and IR measurements and has been shown to be the same in dominant versus nondominant limbs and should be approximately $180^{\circ} .49$

The criteria to progress from Phase III requires the athlete to have a strength limb symmetry index (LSI) of $80 \%$ or greater at external rotation at $0^{\circ}$ abduction (ER0), internal rotation at $0^{\circ}$ abduction (IR0), abduction and scaption at $90^{\circ}$ and belly press. It is important to assess belly press as this isolates the subscapularis muscle and provides a good indication of recovery following the violation of the muscle during surgery. ${ }^{50}$ In addition, assessing periscapular muscular strength should be performed if the scapular dyskinesis test is abnormal. This can easily be assessed with the use of a handheld dynamometer (HHD) which offers an accurate, reliable means of measuring isometric muscular strength in multiple positions.

It is important to consider the athletes psychological readiness to return to sport. Evaluating if the athlete is mentally prepared for higher level activity and eventual return to sport should be considered. Outcome measures such as the Athlete Fear Avoidance Questionnaire (AFAQ) specifically targets athletes on their thoughts about their injuries and returning to play. ${ }^{51}$ It could be used to identify potential psychological barriers to rehabilitation. If an athlete is struggling with this, it is important to refer to a sports psychologist, as this could be the biggest issue holding back an athlete and if not addressed now, the athlete may be delayed with their return to full participation once they have completed the final Phase of rehabilitation.

If the individual is involved in a sport that does not require power movements, involve contact, or overhead sport demands, once Phase III criteria have been achieved, they can be discharged from therapy with a maintenance program. If their sport does require these demands, they will advance to Phase IV of the rehabilitation program once their strength values are $>90 \%$ of their contralateral limb. Strength must also be tested at external rotation at $90^{\circ} \mathrm{ab}-$ duction (ER90) and internal rotation at $90^{\circ}$ abduction (IR90) so the ER/IR ratio can be formulated. The balance between external and internal rotation strength is important to normal glenohumeral function. An adequate external-internal

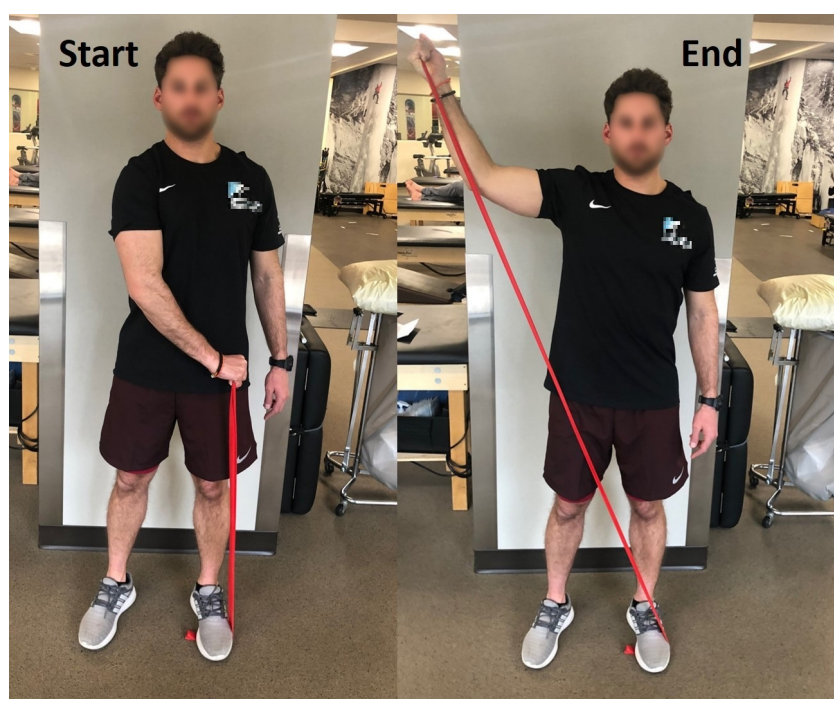

Figure 4. PNF D2 movement pattern with resistance. The movement pattern is diagonal and spiral in nature and crosses midline.

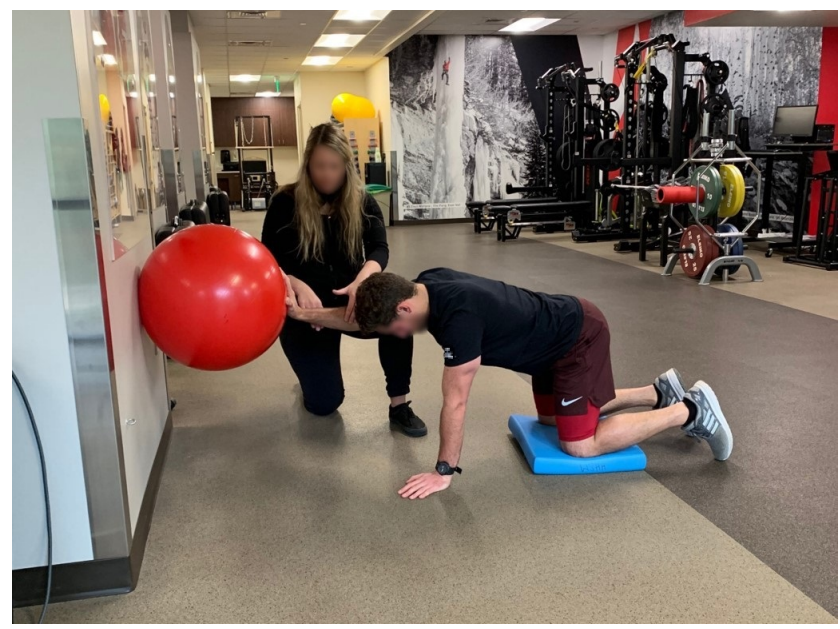

Figure 5. Rhythmic stabilization in quadruped position. The right limb is in an overhead, closed chain position.

rotator muscle strength ratio at $90^{\circ}$ abduction has been emphasized in the literature with a minimum of $65 \%$ but optimal between $66 \%$ and $75 \% .{ }^{52,53}$

\section{PHASE IV: POWER PHASE}

The main goal of this phase of rehabilitation is to maximize power development. Power equals force times velocity. Since force production was the biggest focus of Phase III, the incorporation of velocity into Phase IV will maximize power generation. Based on available evidence, the following guidelines for dosage are recommended when designing a power program for the shoulder. ${ }^{31,43,54,55}$ The athlete should work at $80-100 \%$ of their 1RM for 3-6 repetitions for 3-6 sets. Functional trainers are cable machines which allow unrestricted multiple planes of motion. They can be a 
good tool to accomplish power production, with the ability to perform basic exercises like the 90/90 ER for power or progressing to a push-pull arm cable exercise which incorporates the entire kinetic chain (Figure 6).

Plyometrics are also an excellent method to assist in the development of power, recruit fast twitch muscle fibers and enhance neuromuscular control. ${ }^{54}$ Plyometric training utilizes the stretch - shortening cycle (SSC) by using a lengthening movement (eccentric) which is quickly followed by a shortening movement (concentric) to produce an explosive reaction. 56

UE plyometric exercises can be performed in both an open kinetic chain (OKC) and CKC position. Progression of UE plyometrics performed in an OKC position begins with two-handed drills such as chest pass, side-to-side throws and overhead soccer throws. Once they are performed successfully, the athlete can progress to one-handed drills such as, standing one-handed arm throws, one-handed side arm throw, 90/90 wall dribbles (Figure 7), and prone ball drops. ${ }^{56,57}$ CKC UE plyometrics can be initiated in a partial weight bearing position by performing wall plyometric push - ups or reformer plyometric UE jumps (Figure 8), progressing to full weight bearing plyometric push - ups, depth drop plyometric push - ups, and clap push - ups. ${ }^{54}$

Criterion to progress from Phase IV is dependent upon the sport the athlete intends to return to. For simplicity this is classified as either overhead athlete or non-overhead athlete (see Appendix 1). Functional testing options for individuals returning to sport following shoulder injury and surgery is significantly limited, compared to lower extremity functional tests. There are few high-level evidence studies and few tests with good psychometric properties, with no gold standard test proposed. The presented criterionbased functional testing is based on clinical experience and expertise. The tests can be divided into UE power and closed chain tests and additional tests for the overhead athlete. Bilateral UE tests are compared to normative values while the goal for unilateral tests is a LSI of $>90 \%$. The UE CKC tests described in the literature include the UE YBAL ${ }^{\mathrm{TM}}$ test and closed kinetic chain upper extremity stability test (CKCUEST). The UE YBAL ${ }^{\mathrm{TM}}$ test, the CKCUEST and modified push up test all assess shoulder stability and endurance and would be beneficial for assessment of return to sport ability in both overhead and non-overhead athletes. It is dependent on the rehabilitation specialist to decide which one is most relevant for the individual athlete. Tests that have normative values to compare the athlete with are more desirable than comparing to the uninvolved UE because decreased function and performance of the uninvolved limb over time or non-dominant limb may produce inflated LSI and misrepresent the functional ability of the rehabilitated UE. The single arm seated shot-put test, which assesses isolated power, can also be utilized if the rehabilitative specialist feels it is relevant to the athlete.

Testing for overhead athletes should also include UE OKC and dynamic stabilization tests such as the 90/90 wall dribble test (WDT) and prone ball drop test, which are beneficial in identifying both scapular and cuff weaknesses and poor endurance. ${ }^{54,57,58}$ The WDT involves using a $2 \mathrm{lb}$ medicine ball and the athlete standing in an athletic stance at the corner of a wall, with their shoulder abducted and exter-

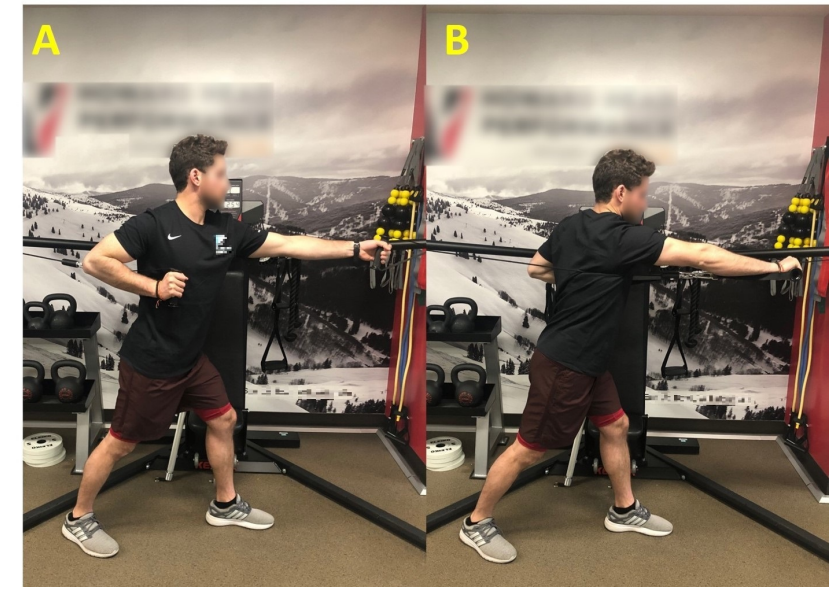

Figure 6A, 6B. Push-pull arm cable exercise.

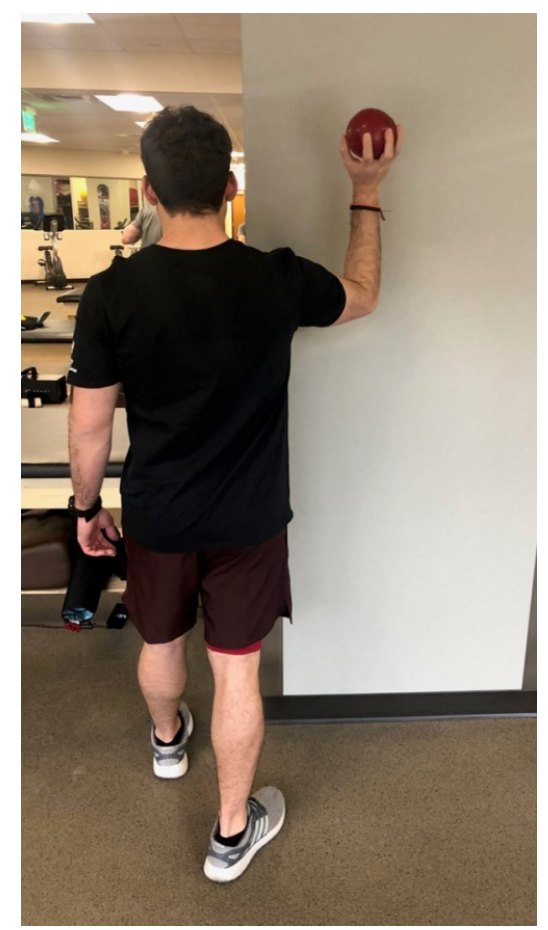

Figure 7. 90/90 Wall dribble performed as an OKC exercise.

nally rotated to $90^{\circ}$. The athlete must perform quick dribbles at $165 \mathrm{bpm}$ (use a metronome) for 60 seconds, maintaining proper position and scapular control, in order to pass. The prone ball drop test involves lying prone on a plinth with the arm in horizontal abduction. The number of releases and catches of a $2 \mathrm{lb}$ medicine ball in 30 seconds is recorded. Comparison between dominant and non-dominant arm is used for passing criteria. ${ }^{59}$

Negrete et al reported that the modified pull up and push up tests may be beneficial assessment tools for throwing athletes given their predictive validity related to throwing distance as compared to other functional tests. ${ }^{60}$ Analysis revealed that the modified pull - up test was the best predictor of a softball throw for distance as it had the high- 
est correlation with the distance thrown $(r=0.70)$, followed closely by the push up test $(r=0.63) .60,61$ Normative values are based on testing of recreational athletes so these goals would be the bare minimum an athlete should aim to achieve.

Reassessment of LE YBAL ${ }^{\mathrm{TM}}$ and FMS ${ }^{\mathrm{TM}}$ at this time frame would also be appropriate in order to see improvements and to be aware of continuing deficits that remain. The goal for LE YBAL ${ }^{\mathrm{TM}}$ is $<4 \mathrm{~cm}$ difference between limbs while the FMS ${ }^{\mathrm{TM}}$ goal is a score of 14 or more.

\section{PHASE V: RETURN TO PLAY PROGRAM}

The phases of rehabilitation which have been discussed in this clinical commentary represent a progression in which exercises become more demanding and the stresses applied to the shoulder joint gradually intensify. After completion of Phase IV, the athlete has demonstrated enough ROM, neuromuscular control, strength and power to start a return to play progression. This Phase will be dependent on the sport or activity that the athlete is returning to and due to the enormous variety, this section will briefly highlight the necessary components to this challenging process.

Regardless of what sport the athlete is returning to, it is essential to know the demands of the specific sport and the demands of the position the athlete plays. For overhead athletes an interval throwing program (ITP) can be introduced during this phase. The ITP gradually introduces quantity, distance, intensity, and types of throws needed to facilitate the restoration of normal throwing motions for the specific throwing sport. ${ }^{62}$ At least six weeks of plyometric training should have been completed prior to starting an ITP. This allows for soft tissue adaptations to occur and prepares the athlete for the stress of throwing.

A graded return to full participation should include practicing the skills needed for the sport, initiating drills without contact, progressing to contact drills, increasing frequency and intensity of training situations to eventually meet the game requirements, with collaboration of the coaches in order to find appropriate progressions. Once the athlete is completing full training sessions which mimic the frequency and intensity of the sport and/or have successfully completed an ITP without pain, while also exhibiting proper throwing mechanics, introducing them to competition situations would be the final step. For overhead athletes an additional outcome measure, the Kerlan-Jobe Orthopaedic Clinic (KJOC) shoulder and elbow score, should also be completed and can be used to gauge readiness to return to full participation with a score of $90 \%$ or higher being needed, prior to returning to competition. ${ }^{63}$

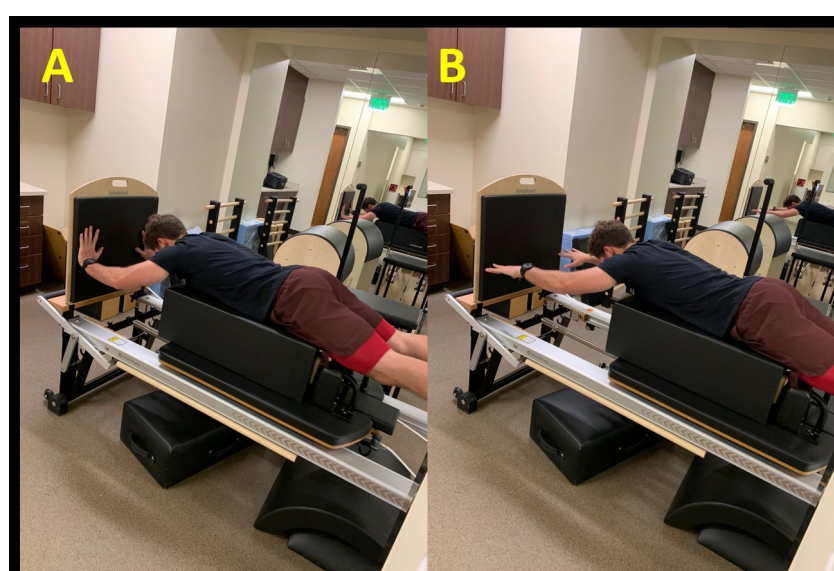

Figure 8. Plyometric UE jumps at start position (A) and end position (B) on Pilates reformer.

\section{CONCLUSION}

This clinical commentary describes a criterion-based rehabilitation protocol following Latarjet procedure in an athletic population. It describes progression through clearly defined phases and recommended criteria to achieve prior to progression into the next phase. Phase I focuses on protection and initiation of PROM with supplemental exercises to address ROM of surrounding joints and active scapular mobility. Phase II focuses on initiating AAROM and AROM, neuromuscular reaction drills, such as RS and JPS, and progression of scapular control. Phase III focuses on advancing ROM, strength including functional and in CKC positions and advancing neuromuscular drills such as RS at end range and PNF. Phase IV introduces power specific exercises and plyometrics starting with two-handed exercises to onehanded. A final reassessment of the full kinetic chain with FMS $^{\mathrm{TM}}$ or LE YBAL ${ }^{\mathrm{TM}}$ is helpful during this Phase. Additional testing at the end of Phase IV is chosen and utilized to see if the athlete is ready for return to play progressions (dependent upon the sport to which they are returning). Functional CKC and OKC tests are recommended and an additional pathway for over-head athletes is used. Phase V focuses on return to full participation with a graded progression, which is dependent on the demands of the specific sport. By methodically following the recommended criterion-driven rehabilitation algorithm and achieving the set criteria, it is anticipated that the athlete will have completed a very thorough rehabilitation program that addresses ROM, neuromuscular control, strength, power and a graded return to play.

Submitted: October 14, 2019 CDT, Accepted: May 17, 2020 CDT 


\section{REFERENCES}

1. Smith TO. Immobilisation following traumatic anterior glenohumeral joint dislocation: A literature review. Injury. 2006;37(3):228-237. doi:10.1016/i.injur y.2005.06.005

2. Owens BD, Duffey ML, Nelson BJ, DeBerardino TM, Taylor DC, Mountcastle SB. The incidence and characteristics of shoulder instability at the United States Military Academy. Am J Sports Med. 2007;35(7):1168-1173. doi:10.1177/036354650629517 $\underline{9}$

3. Waterman B, Owens BD, Tokish JM. Anterior shoulder instability in the military athlete. Sports Health. 2016;8(6):514-519. doi:10.1177/19417381166 $\underline{72161}$

4. Owens BD, Agel J, Mountcastle SB, Cameron KL, Nelson BJ. Incidence of glenohumeral instability in collegiate athletics. Am J Sports Med. 2009;37(9):1750-1754. doi:10.1177/036354650933459 1

5. Dickens JF, Rue J-P, Cameron KL, et al. Successful return to sport after arthroscopic shoulder stabilization versus nonoperative management in contact athletes with anterior shoulder instability: A prospective multicenter study. Am I Sports Med. 2017;45(11):2540-2546. doi:10.1177/03635465177125 $\underline{05}$

6. Phadnis J, Arnold C, Elmorsy A, Flannery M. Utility of the instability severity index score in predicting failure after arthroscopic anterior stabilization of the shoulder. Am J Sports Med. 2015;43(8):1983-1988. do i:10.1177/0363546515587083

7. Shaha JS, Cook JB, Song DJ, et al. Redefining "Critical” Bone Loss in Shoulder Instability: functional outcomes worsen with "subcritical" bone loss. Am J Sports Med. 2015;43(7):1719-1725. doi:10.1 $177 / 0363546515578250$

8. Beranger JS, Klouche S, Bauer T, Demoures T, Hardy P. Anterior shoulder stabilization by BristowLatarjet procedure in athletes: Return-to-sport and functional outcomes at minimum 2-year follow-up. Eur J Orthop Surg Traumatol. 2016;26(3):277-282. do $\mathrm{i}: 10.1007 / \mathrm{s} 00590-016-1751-5$

9. Cerciello S, Corona K, Morris BJ, Santagada DA, Maccauro G. Early outcomes and perioperative complications of the arthroscopic Latarjet procedure: Systematic review and meta-analysis. Am J Sports Med. 2019;47(9):2232-2241. doi:10.1177/0363546518 $\underline{783743}$
10. Hurley ET, Jamal MS, Ali ZS, Montgomery C, Pauzenberger L, Mullett H. Long-term outcomes of the Latarjet procedure for anterior shoulder instability: A systematic review of studies at 10-year follow-up. J Shoulder Elbow Surg. 2019;28(2):e33-e39. doi:10.1016/i.jse.2018.08.028

11. Privitera DM, Sinz NJ, Miller LR, et al. Clinical outcomes following the Latarjet procedure in contact and collision athletes. J Bone Joint Surg Am. 2018;100(6):459-465. doi:10.2106/jbjs.17.00566

12. Rollick NC, Ono Y, Kurji HM, et al. Long-term outcomes of the Bankart and Latarjet repairs: A systematic review. Open Access J Sports Med. 2017;8:97-105. doi:10.2147/oajsm.s106983

13. Young AA, Maia R, Berhouet J, Walch G. Open Latarjet procedure for management of bone loss in anterior instability of the glenohumeral joint. $J$ Shoulder Elbow Surg. 2011;20(2 Suppl):S61-S69. doi:1 0.1016/j.jse.2010.07.022

14. Allain J, Goutallier D, Glorion C. Long-Term Results of the Latarjet Procedure for the Treatment of Anterior Instability of the Shoulder. J Bone Joint Surg Am. 1998;80(6):841-852. doi:10.2106/00004623-1998 06000-00008

15. Hovelius LK, Sandström BC, Rösmark DL, Saebö M, Sundgren KH, Malmqvist BG. Long-term results with the Bankart and Bristow-Latarjet procedures: Recurrent shoulder instability and arthropathy. $J$ Shoulder Elbow Surg. 2001;10(5):445-452. doi:10.106 $\underline{7 / \mathrm{mse} .2001 .117128}$

16. Singer GC, Kirkland PM, Emery RJ. Coracoid transposition for recurrent anterior instability of the shoulder. A 20-year follow-up study. J Bone Joint Surg $B r .1995$;77(1):73-76. doi:10.1302/0301-620x.77b1.78 $\underline{22401}$

17. Kee YM, Kim HJ, Kim JY, Rhee YG. Glenohumeral arthritis after Latarjet procedure: Progression and it's clinical significance. J Orthop Sci. 2017;22(5):846-851. doi:10.1016/i.jos.2017.06.008

18. Gaunt BW, Shaffer MA, Sauers EL, Michener LA, McCluskey GMI, Thigpen CA. The American Society of Shoulder and Elbow Therapists' consensus rehabilitation guideline for arthroscopic anterior capsulolabral repair of the shoulder. J Orthop Sports Phys Ther. 2010;40(3):155-168. doi:10.2519/jospt.201 $\underline{0.3186}$ 
19. Cuéllar R, Ruiz-Ibán MA, Cuéllar A. Anatomy and biomechanics of the unstable shoulder. Open Orthop J. 2017;11(1):919-933. doi:10.2174/187432500171101 $\underline{0919}$

20. Soslowsky LJ, Flatow EL, Bigliani LU, Mow VC. Articular geometry of the glenohumeral joint. Clin Orthop Relat Res. 1992;(285):181-190. doi:10.1097/00 003086-199212000-00023

21. Soslowsky LJ, Flatow EL, Bigliani LU, Pawluk RJ, Ateshian GA, Mow VC. Quantitation of in situ contact areas at the glenohumeral joint: A biomechanical study. J Orthop Res. 1992;10(4):524-534. doi:10.1002/i or.1100100407

22. McCluskey GM, Getz BA. Pathophysiology of anterior shoulder instability. J Athl Train. 2000;35(3):268-272.

23. Latarjet M. Treatment of recurrent dislocation of the shoulder. Lyon Chir. 1954;49(8):994-997.

24. Patte D, Debeyre J. Luxations récidivantes de l'épaule. Encycl Med Chir. Elsevier, Paris, ed. Tech Chir Orthop. 1982:44-265.

25. Lacheta L, Siebenlist S, Imhoff AB, Willinger L. Recurrent instability and instability arthropathy. Unfallchirurg. 2018;121(2):142-151. doi:10.1007/s001 $\underline{13-017-0408-0}$

26. Payne WB, Kleiner MT, McGarry MH, Tibone JE, Lee TQ. Biomechanical comparison of the Latarjet procedure with and without a coracoid bone block. Knee Surg Sports Traumatol Arthrosc. 2016;24(2):513-520. doi:10.1007/s00167-015-3885-0

27. Wellmann M, Petersen W, Zantop T, et al. Open shoulder repair of osseous glenoid defects:

Biomechanical effectiveness of the Latarjet procedure versus a contoured structural bone graft. Am J Sports Med. 2009;37(1):87-94. doi:10.1177/03635465083267 $\underline{14}$

28. Yamamoto N, Muraki T, An K-N, et al. The stabilizing mechanism of the Latarjet procedure: A cadaveric study. J Bone Joint Surg Am. 2013;95(15):1390-1397. doi:10.2106/jbjs.1.00777

29. Itoi E, Hatakeyama Y, Urayama M, Pradhan RL, Kido T, Sato K. Position of immobilization after dislocation of the shoulder. A cadaveric study. J Bone Joint Surg Am. 1999;81(3):385-390. doi:10.2106/00004 623-199903000-00011

30. Popchak A, Patterson-Lynch B, Christain H, Irrgang JJ. Rehabilitation and return to sports after anterior shoulder stabilization. Ann Joint. 2017;2(10):62-62. doi:10.21037/aoj.2017.10.06
31. Kraemer WJ, Ratamess NA, French DN. Resistance training for health and performance. Curr Sports Med Rep. 2002;1(3):165-171. doi:10.1249/00149619-20020 6000-00007

32. Ellenbecker TS, Cools A. Rehabilitation of shoulder impingement syndrome and rotator cuff injuries: An evidence-based review. Br J Sports Med. 2010;44(5):319-327. doi:10.1136/bjsm.2009.058875

33. van der Zwaag HM, Brand R, Obermann WR, Rozing PM. Glenohumeral osteoarthrosis after PuttiPlatt repair. J Shoulder Elbow Surg. 1999;8(3):252-258. doi:10.1016/s1058-2746(99)90138-6

34. Myers JB, Lephart SM. The role of the sensorimotor system in the athletic shoulder. J Athl Train. 2000;35(3):351-363.

35. Zuckerman JD, Gallagher MA, Cuomo F, Rokito A. The effect of instability and subsequent anterior shoulder repair on proprioceptive ability. J Shoulder Elbow Surg. 2003;12(2):105-109. doi:10.1067/mse.200 $\underline{3.4}$

36. Dover G, Powers ME. Reliability of joint position sense and force-reproduction measures during internal and external rotation of the shoulder. J Athl Train. 2003;38(4):304-310.

37. Davies GJ, Dickoff-Hoffman S. Neuromuscular testing and rehabilitation of the shoulder complex. $J$ Orthop Sports Phys Ther. 1993;18(2):449-458. doi:10.2 519/jospt.1993.18.2.449

38. Reinold MM, Gill TJ, Wilk KE, Andrews JR. Current concepts in the evaluation and treatment of the shoulder in overhead throwing athletes, part 2: Injury prevention and treatment. Sports Health. 2010;2(2):101-115. doi:10.1177/1941738110362518

39. Speer KP, Cavanaugh JT, Warren RF, Day L, Wickiewicz TL. A role for hydrotherapy in shoulder rehabilitation. Am J Sports Med. 1993;21(6):850-853. doi:10.1177/036354659302100616

40. Cricchio M, Frazer C. Scapulothoracic and scapulohumeral exercises: A narrative review of electromyographic studies. J Hand Ther. 2011;24(4):322-334. doi:10.1016/j.jht.2011.06.001

41. McClure P, Tate AR, Kareha S, Irwin D, Zlupko E. A Clinical Method for Identifying Scapular Dyskinesis, Part 1: Reliability. J Athl Train. 2009;44(2):160-164. doi:10.4085/1062-6050-44.2.160

42. Schoenfeld BJ. Potential mechanisms for a role of metabolic stress in hypertrophic adaptations to resistance training. Sports Med. 2013;43(3):179-194. $\underline{\mathrm{d}}$ oi:10.1007/s40279-013-0017-1 
43. American College of Sports Medicine. American College of Sports Medicine position stand.Progression models in resistance training for healthy adults. Med Sci Sports Exerc.

2009;41(3):687-708. doi:10.1249/mss.0b013e3181915 $\underline{670}$

44. De Mey K, Danneels L, Cagnie B, Cools A. Are kinetic chain rowing exercises relevant in shoulder and trunk injury prevention training? Br J Sports Med. 2011;45(4):320-320. doi:10.1136/bjsm.2011.084038.3 $\underline{0}$

45. Jaggi A, Lambert S. Rehabilitation for shoulder instability. Br J Sports Med. 2010;44(5):333-340. doi:1 $\underline{0.1136 / \text { bjsm.2009.059311 }}$

46. Ellenbecker TS. Shoulder Rehabilitation: NonOperative Treatment. Thieme Medical Publishers; 2006. doi:10.1055/b-002-52052

47. Ju S-K, Yoo W-G. Electromyography activity of the deltoid muscle of the weight-bearing side during shoulder flexion in various weight-bearing positions. J Phys Ther Sci. 2015;27(10):3285-3286. doi:10.1589/i pts.27.3285

48. Kibler BW, McMullen J. Scapular dyskinesis and its relation to shoulder pain. J Am Acad Orthop Surg. 2003;11(2):142-151. doi:10.5435/00124635-20030300 $\underline{0-00008}$

49. Wilk KE, Macrina LC, Arrigo C. Passive range of motion characteristics in the overhead baseball pitcher and their implications for rehabilitation. Clin Orthop Relat Res. 2012;470(6):1586-1594. doi:10.100 7/s11999-012-2265-z

50. Tokish JM, Decker MJ, Ellis HB, Torry MR, Hawkins RJ. The belly-press test for the physical examination of the subscapularis muscle: Electromyographic validation and comparison to the lift-off test. J Shoulder Elbow Surg. 2003;12(5):427-430. doi:10.1016/s1058-2746(03)0004 $\underline{7-8}$

51. Dover G, Amar V. Development and validation of the athlete fear avoidance questionnaire. J Athl Train. 2015;50(6):634-642. doi:10.4085/1062-6050-49.3.75

52. Byram IR, Bushnell BD, Dugger K, Charron K, Harrell FEJ, Noonan TJ. Preseason shoulder strength measurements in professional baseball pitchers: Identifying players at risk for injury. Am J Sports Med. 2010;38(7):1375-1382. doi:10.1177/036354650936040 $\underline{4}$
53. Ellenbecker T, Roetert EP. Age specific isokinetic glenohumeral internal and external rotation strength in elite junior tennis players. J Sci Med Sport. 2003;6(1):63-70. doi:10.1016/s1440-2440(03)80009-9

54. Davies G, Riemann BL, Manske R. Current concepts of plyometric exercise. Int J Sports Phys Ther. 2015;10(6):760-786.

55. Haff GG, Triplett NT. Essentials of Strength Training and Conditioning. 4th ed. Champaign, IL: Human Kinetics; 2016.

56. Kraemer WJ, Newton RU. Training for muscular power. Phys Med Rehabil Clin N Am. 2000;11(2):341-368, vii. doi:10.1016/s1047-9651(18)3 $\underline{0133-5}$

57. Wilk KE, Arrigo C. Current concepts in the rehabilitation of the athletic shoulder. J Orthop Sports Phys Ther. 1993;18(1):365-378. doi:10.2519/jospt.199 3.18.1.365

58. Kovacs MS, Roetert EP, Ellenbecker TS. Efficient deceleration: The forgotten factor in tennis-specific training. Strength Cond J. 2008;30(6):58-69. doi:10.15 19/ssc.0b013e31818e 5fbc

59. Wilk KE, Arrigo CA, Hooks TR, Andrews JR. Rehabilitation of the overhead throwing athlete: There is more to it than just external rotation/ internal rotation strengthening. Pm r. 2016;8(3 Suppl):S78-S90. doi:10.1016/i.pmri.2015.12.005

60. Negrete RJ, Hanney WJ, Kolber MJ, et al. Reliability, minimal detectable change, and normative values for tests of upper extremity function and power. J Strength Cond Res. 2010;24(12):3318-3325. doi:10.1519/jsc.0b013e3181e $\underline{7259 \mathrm{c}}$

61. Negrete RJ, Hanney WJ, Kolber MJ, et al. Can upper extremity functional tests predict the softball throw for distance: A predictive validity investigation. Int J Sports Phys Ther. 2011;6(2):104-111.

62. Reinold MM, Wilk KE, Reed J, Crenshaw K, Andrews JR. Interval sport programs: Guidelines for baseball, tennis, and golf. J Orthop Sports Phys Ther. 2002;32(6):293-298. doi:10.2519/jospt.2002.32.6.293

63. Alberta FG, El Attrache NS, Bissell S, et al. The development and validation of a functional assessment tool for the upper extremity in the overhead athlete. Am J Sports Med. 2010;38(5):903-911. doi:10.1177/0363546509355642 


\section{SUPPLEMENTARY MATERIALS}

\section{Appendix 1}

Download: https://ijspt.scholasticahq.com/article/18707-latarjet-procedure-for-the-treatment-of-anteriorglenohumeral-instability-in-the-athlete-key-considerations-for-rehabilitation/attachment/49307.docx 